\title{
Noise Constraints Effecting Optimal Propeller Designs
}

Christopher J. Miller

Lewis Research Center

Cleveland, Ohio

and

John P. Sullivan

Purdue University

West Lafayette, Indiana

Prepared for the

SAE General Aviation Aircraft Meeting and Exposition sponsored by the Society of Automotive Engineers

Wichita, Kansas, April 16-19, 1985 


\section{ABS TRACT}

A preliminary design tool for advanced propellers has been developed combining a fast vortex lattice aerodynamic analysis, a fast subsonic point source noise analysis, and an optimization scheme using a conjugate directions $\underset{f}{f}$ method. Twist, chord and sweep distributions $\underset{1}{ }$ are optimized to simultaneously improve both the aerodynamic performance and the noise observed at a fixed relative position.

The optimal noise/performance trade-offs for straight and advanced concept blades are presented. The techniques used include increasing the blade number, blade sweep, reducing the rotational speed, shifting the spanwise loading and diameter changes. Some methods yield improvement in both efficiency and noise.

\section{INTRODUCTION}

PREVIOUS WORK $(\underline{1}, \underline{2}) *$ HAS IDENTIFIED THE performance gains and noise reductions available through the use of advanced concept propeller blades. These works optimized the performance only; noise was considered as a separate issue. To properly investigate advanced propeller concepts as well as conventional geometries, a design tool should be able to optimize both performance and noise at the same time. This paper describes the development of such a design tool and the noise/performance trade-offs available from optimal geometries.

The design program consists of three parts: (1) a vortex lattice method for the aerodynamic performance prediction; (2) a subsonic compact source model in the time domain for the noise prediction; and ( 3 ) an optimization scheme using a conjugate directions method.

*Numbers in parentheses designate references at end of paper.

\section{AERODYNAMIC ANALYSIS}

The vortex lattice method has been used by various researchers ( 2 to $\underline{7}$ ) in the study of propeller performance. The basic calculation geometry is shown in Fig. 1. There is a single curved lifting line divided spanwise into a set of horseshoe vortices. This lifting line is a long the quarter-chord line of the blade. At control points on the three-quarter chord line, a boundary condition of no flow through the mean camber surface is imposed. The trailing vortices representing the wake are prescribed to lie on the surface swept out by the quarterchord line with pitch defined by the advance ratio. This method is a steady symetric analysis, so the radial circulation distribution is the same for all blades.

The equations to be solved are based on the boundary condition that there is no flow through the chord plane surface at the control points. The boundary condition for the $j^{\text {th }}$ vortex lattice element is therefore:

$$
\vec{v}_{j}^{c} \cdot \hat{n}_{j}=\left[\vec{v}_{\text {inflow }_{j}}+\vec{r}_{j}^{c} \overrightarrow{x \vec{s}}+\vec{v}_{j}\right] \cdot \hat{n}_{j}=0
$$

where

$$
\begin{array}{ll}
\vec{V}_{j}^{c} & \text { total velocity at } \vec{r}_{j}^{c} \\
\hat{n}_{j} & \begin{array}{l}
\text { normal to the chord plane at the con- } \\
\text { trol point }
\end{array} \\
\vec{V}_{\text {inflow }} & \begin{array}{l}
\text { axisymmetric flow field due to the hub } \\
\vec{r}_{j}^{c}
\end{array} \\
\vec{r}_{j}^{c} & \text { control point position } \\
\overrightarrow{v i} & \text { shaft speed and axis [radians/sec] } \\
\vec{v}_{j} & \text { total induced velocity at } \vec{r}_{j}^{c}
\end{array}
$$


The Biot-Savart Law is used to calculate the induced velocities due to the horseshoe vorticies. The induced velocity at the control point of lattice element $j$ of blade 1 due to the vortex of element $i$ of blade $k$ is given byт

$$
\vec{v}_{i j k}=\frac{-r_{i}}{4 \pi R_{t i p}} \int \frac{\vec{R}_{i j k} x \vec{d}_{i k}}{1 \vec{R}_{i j k} \mid 1^{3}}
$$

where

$\Gamma_{i} \quad$ circulation strength of horseshoe vortex $i$ per unit length

$\vec{R}_{i j k} \vec{r}_{j}^{c}-$ position of $\vec{d}_{i k}$

$\mathrm{R}_{\text {tip }}$ propeller tip radius

$\vec{d} \lambda_{i k}$ differential segment of the horseshoe vortex.

Since the circulations are the unknowns, the boundary condition provides a set of simultaneous equations for the circulations.

Once the circulations have been determined, the forces on each element are calculated using the Kutta-Joukowski law. These forces are summed to provide the overall forces on the propeller. The blade forces for the $j$ th element are given by:

$$
\vec{F}_{j}=\rho_{o} s \vec{V}_{j}^{m} \times \vec{\Gamma}_{j}+D_{v} \frac{\vec{V}_{j}^{m}}{1 \vec{V}_{j}^{m} \mid 1}
$$

\section{where}

o density

$s$ bound vortex length

$\vec{r}_{j} j^{\text {th }}$ bound vortex segment circulation

$\vec{v}_{j}^{u \prime} \quad$ total velocity at the bound segment midpoint

${ }_{\mathrm{V}}^{\mathrm{V}}$ viscous drag estimate.

Here the viscous drag is calculated based on the section lift coefficient of the element, $C_{\ddot{x}}:$

$$
\begin{gathered}
C_{d}=C_{d o}+K\left(C_{l}-C_{l}^{\text {design }}\right)^{2} \\
D_{v}=\frac{1}{2} \rho_{o} s c\left(v_{j}^{M} \cdot v_{j}^{M}\right)
\end{gathered}
$$

\author{
$\mathrm{C}_{\text {do }} \quad$ zero lift drag coefficient

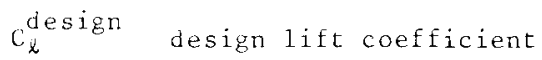 \\ c chord length \\ K drag polar constant.
}

As a verification, Figs. 2 to 5 compare the predicted results with experimental test data. The data in Figs. 2 and 3 are for a representative general aviation propeller tested at the NASA Lewis kesearch Center (8). The data in Figs. 4 and 5, taken from Ref. (9), are for a quarter scale general aviation propeller modeled after the biade on a Cessna 172: the McCauley 1c160. In both cases, the predicted performance agrees well, but the predicted variation of power coefficient $\left(C_{p}\right)$ with advance ratio $(J)$ has more curvature than the experimental results. The fixed wake model is partially to blame: the induced velocities would increase the wake helix angle, and hence would increase the induced drag at the higher loadings. This factor would raise the high loading prediction of $C_{P}$ and straighten the curve.

In these figures, the following symbols are used

B blade number

$B_{3 / 4}$ blade pitch at the $3 / 4$ radius

J $\quad V / n D=$ advance ratio

$\mathrm{V}$ axial inflow velocity (flight velocity)

A $V / a k=J / \pi=$ alternate advance ratio

$\mathrm{C}_{\mathrm{T}} \quad \mathrm{\Gamma} /\left(\rho_{\mathrm{o}} \mathrm{n}^{2} \mathrm{D}^{4}\right)=$ thrust coefficient

$C_{P} \quad P /\left(p_{0} n^{3} D^{5}\right)=$ power coefficient.

\section{NOISE ANALYSIS}

The noise analysis method is based on the work of Lowson (10). This analys is assumes that there is no mean flow (static operation) and that the continuous distribution of noise sources on the blade can be represented by point sources. These assumptions are met when considering low speed operation, as in take-off and climb, such that the helical tip speeds are less than about Mach 0.75 .

Lowson did not consider the noise produced by a radial force in his analysis. So, an additional term for the radial force noise has been derived for use in this study. The equation which must be solved for the normalized pressure perturbation produced by the propeller is:

where

$\mathrm{C}_{d} \quad$ viscous drag coefficient 


$$
\begin{aligned}
& \frac{p^{\prime}}{p_{0}}=\left[\frac{\gamma M_{\Omega}^{2} R_{t i p}^{2}}{\pi^{3}\left(1-M_{r}\right)^{2} r^{2}}\right] \\
& \begin{array}{l}
\cdot\left[\Delta C_{T}\left\{\frac{-\left(x-x_{s}\right)}{l-M_{r}}\left(\frac{1}{a_{0}} \frac{\partial M_{r}}{a_{t}}+\frac{1-M_{s}^{2}}{r}\right)\right)\right. \\
+\Delta C_{\theta} \frac{R_{t i p}}{\pi R_{s}}\left\{\frac{\Omega}{a_{0}}\left(-r_{o b s} \sin (\phi) \cos (\theta)\right)\right.
\end{array} \\
& \left.+\frac{-r_{o b s} \sin (\phi) \sin (\theta)}{1-M_{r}}\left(\frac{1}{a_{0}} \frac{\partial M_{r}}{\partial t}+\frac{1-M_{s}^{2}}{r}\right)\right) \\
& +\Delta C_{R}\left\{\frac{\Omega}{a_{0}}\left(r_{o b s} \sin (\phi) \sin (\theta)\right)\right. \\
& \left.+\frac{R_{s}-r_{o b s} \sin (\phi) \sin (\theta)}{1-M_{r}}\left(\frac{1}{a_{0}} \frac{\partial M}{\partial t}+\frac{1-M_{s}^{2}}{r}\right)\right) \\
& \left.+\Delta K_{q}\left\{\frac{r}{1-M_{r}}\left(\frac{1}{a_{0}} \frac{\partial M_{r}}{\partial t}+\frac{M_{r}-M_{s}^{2}}{r}\right)\right)\right]_{r e t}
\end{aligned}
$$

\begin{tabular}{|c|c|}
\hline$a_{0}$ & speed of sound \\
\hline$p_{o}$ & ambient pressure \\
\hline $\mathrm{M}_{32}$ & $\operatorname{sir} / \mathrm{a}_{0}$ \\
\hline $\mathrm{M}_{\mathrm{r}}$ & $-M_{s}\left(r_{o b s} / r\right) \sin (\phi) \sin (\theta)$ \\
\hline $\mathrm{M}_{\mathrm{s}}$ & $s_{\mathrm{s}} / \mathrm{a}_{0}$ \\
\hline$r$ & ||$r_{i}||=|| x_{i}-y_{i}||$ \\
\hline$x_{i}$ & $\begin{array}{l}(X, Y, 0)=\left(r_{\text {obs }} \cos (\phi), r_{o b s} \sin (\phi),\right. \\
0)=\text { observer position }\end{array}$ \\
\hline$y_{i}$ & $\begin{array}{l}\left(X_{S}, R_{S} \cos (\theta), R_{S} \sin (\theta)\right)=\text { source } \\
\text { position }\end{array}$ \\
\hline$\Delta \mathrm{C}_{\mathrm{T}}, \Delta \mathrm{C}_{\theta}, \Delta \mathrm{C}_{\mathrm{R}}$ & force coefficients on an element \\
\hline$\Delta \mathrm{K}_{\mathrm{q}}$ & $\begin{array}{l}\text { mass } f 1 \text { ux as force term }=a_{o} q_{m} / \\
\rho_{o}{ }^{2} D^{4}\end{array}$ \\
\hline$q_{\text {In }}$ & volume flux \\
\hline n & shaft speed [rps/s] \\
\hline D & propeller diameter \\
\hline Y & specific heat ratio $=1.4$ \\
\hline$\theta$ & azimuthal angle \\
\hline
\end{tabular}

where
For the noise analysis, the $x$-axis is the forward axis of rotation ( $x$ ). This equation is evaluated at the normalized retarded time $\theta$, for the observer time determined by:

$$
\hat{\mathrm{t}}=\left(\frac{\Delta}{2 \pi}\right) \mathrm{t}=\frac{1}{2 \pi}\left\{\theta+\frac{\Delta}{a_{0}} r(\theta)-\Delta \theta\right\}
$$

where

$\Delta \theta^{s}$ sweep introduced delay.

The propeller is modeled as a distribution of point noise sources with strengths proportional to the local loading and thickness. Fach of the vortex lattice elements is modeled as two noise elements in the spanwise direction (or along the hub to hub quarter-chord line for the bi-blade) to improve the assumption of compactness. The layout of noise sources is shown in Fig. 6 .

The force coefficients, $\left(\Delta \mathrm{C}_{\mathrm{T}}, \Delta \mathrm{C}_{\theta}, \Delta \mathrm{C}_{\mathrm{R}}\right)$, on the noise elements are half of the coefficient on the parent aerodynamic element, and the thickness source term is written in the form of a force coefficient $\left(\Delta \mathrm{K}_{\mathrm{q}}\right)$ for calculation ease. The strength and positions of the source - sink pair used to represent thickness are chosen so that the Rankine body produced has the same chord as the local blade section, and the same frontal area as the noise element.

To increase the execution speed, the pressure perturbation (Eq. (6)) is calculated at equally spaced points in retarded time, $\theta$, along with the associated observer times, $t$. These pressures are then interpolated to an equally spaced set in observer time. The advantage of this method, due to Munro (11), is that the time consuming solution for the retarded time associated with a given observer time is eliminated. Also, the distribution of points in observer time is densest in the region of the pressure peaks which results in an accurate interpolation of the peaks. The multiple blade waveform is created from this single blade waveform by superposition with the proper phase delays.

Once the perturbation pressure time history has been produced, a Fast Fourier Transform is used to obtain the harmonic magnitudes. The overall sound pressure level (OASPL) noise measure is then calculated from these harmonics both in $d B$ and using the $A$-weighted scale for $d B A$.

Figure 7 compares the predicted and measured near field noise, one tip radius away in the plane of rotation for the quarter scale propeller test data in Refs. (9) and (12) to (14). There is a phase shift between the prediction and the experimental data, but the waveform shape and magnitude are in excellent agreement. Additional data is available both ahead of and behind the plane of rotation. These data are compared with predictions in Ref. (15). The comparisons show the same phase shift along with a small magnitude difference. This magnitude 
difference results in a few tenths of a dB difference in the OASPL.

\section{OPTIMIZATION METHOD}

An optimization method automates the process of choosing values of the independent variables to create a "best" result. In this work, the independent variables are the twist, chord, and sweep distributions, while the measure of the result is called the Index of Performance. The Index of Performance (IP) contains both the aerodynamic performance and noise of the propeller and is formulated so that an optimum geometry corresponds to a minimum of the function. Different optimal geonetries are obtained by changing the relative importance of noise versus efficiency in the IP.

The conjugate directions method used for the optimization is that of Powell (16) as modified by Langwill (17). After searching along each of the current set of search directions, the technique searches along the direction resulting from the end points of the intermediate steps. This composite direction is the candidate conjugate direction. If the search proves truitful, the conjugate direction replaces one of the previous search directions.

The line searches are done using a quadratic fit to three function evaluations. If the estimated minimum lies outside the fit interval, the interval is shifted towards the minimum until the minimum is bracketed. Then, if the prediction for the value of the objective function does not agree with the computed value, the fit interval width may be reduced. The minimum interval width allowable is based on the rela$t$ ive magnitude of the random portion of the objective function. This method is rapid and provides some immunity to numerical noise in the objective function.

The Index of Performance (IP) incorporates the constraints in the manner of the SUMT formulation developed by Fiacco and McCormick (18):

$$
\begin{aligned}
I P & =a\left(-c_{T}\right)+(1-a) b N+\frac{1}{w_{c}}\left(C_{p}-c_{p} \text { target }\right)^{2} \\
& +w_{s}^{a}\left[\ln \left(s^{1 i m i t}-s^{I}\right)+w_{s}^{b} s^{I I}\right] \\
& +w_{s I}\left[\sum_{i=1}^{n e l s}(\Gamma(i)+\Gamma(\text { nels }-i))^{2}\right],
\end{aligned}
$$

where

$\begin{array}{ll}C_{\mathrm{T}} & \text { propeller thrust coefficient } \\ \mathrm{C}_{\mathrm{P}} & \text { propeller power coefficient } \\ \mathrm{C}_{\mathrm{p}} \text { arget } & \text { parameter to obtain the desired } \mathrm{C}_{\mathrm{P}} \\ \mathrm{N} & \text { OASPL noise measure in }(\mathrm{d} B) \text { or }(\mathrm{dBA}) \\ \mathrm{s} \text { limit } & \text { limit value of total sweep }\end{array}$

$\Gamma(i)$ blade element circulations

the relative importance of efficiency versus noise

b normalization for noise

nels number of blade elements

$w_{C} \quad C_{p}$ weighting

$W_{S}^{a} \quad$ weight on sweep

$W_{S}^{b} \quad$ weight on sweep "kink" constraint

Whe weight to force bi-blade symmetric loading.

The measure of sweep, $\mathrm{S}^{\mathrm{I}}$, is given by

$$
s^{I}=\sum_{i=1}^{n e l s} \tan ^{-1}\left(\frac{y_{i}^{N}}{x_{i}^{N}}\right)
$$

and the measure of inter-element angle is

$$
S^{I I}=\sum_{i=1}^{n e l s} \cos ^{-1}\left(\frac{t_{i}^{N} \cdot t_{i+1}^{N}}{11 t_{i}^{N}|1| 1 t_{i+1}^{N} \mid 1}\right) \text {, }
$$

where

$$
\begin{aligned}
& x_{i}^{N}, y_{i}^{N} \quad i^{\text {th }} \text { node point coordinates } \\
& \hat{t} j \quad \begin{array}{l}
N \text { tangent to the bound segment between } \\
\text { nodes } j \text { and } j+1 .
\end{array}
\end{aligned}
$$

Because of the numerical noise present in the calculation, the weightings in (8) are not decreased to zero as would be customary. Instead, the weightings are held constant and the offset target values are adjusted. This prevents the amplification of the random noise in the IP.

To further speed the optimization, the scheme is closely coupled with the aerodynamic analysis routine. This is done by recalculating only those portions of the model affected by geometry changes. For example, if an element is retwisted, only the induced velocities at the control point change. The induced velocities at the bound segment and all induced velocities on the other elements remain the same and are reused to calculate the performance. This is most beneficial initially when the search directions correspond to single geometric quantities. Compared with a complete solution for a design state, this technique is 2 to 24 times faster for evaluations of a 12 element blade model. An initial test case for the optimization method was to maximize straight blade ideal 
efficiency as a function of the twist distribution. Goldstein's circulation distribution (19), which yields the maximum ideal efficiency under these assumptions, was obtained, verifying the method.

For a more realistic prediction, a viscous drag estimate is included. This estimate assumes a laminar flow section operating in the laminar bucket with the same dependence of $C_{d o}$ on section thickness as for a Clark-Y airfoil (to be conservative). The $\mathrm{C}_{\ell}$ for stall was chosen to be 1.2 , and stall was modeled by a sharp increase in the section drag coefficient. This drag model is justified because given the design lift coefficient, the corresponding airfoil shape can be found. By including viscous effects, the chord distribution can be a design variable in addition to the twist distribution.

To investigate the trade-offs involved when noise is considered, various techniques for noise reduction are applied to a straight, a propleted and a bi-bladed propeller. These techniques include: reducing the diameter to reduce tip speed, shifting the loading inboard, and reducing the rotational rate to reduce tip speed. For these trade-offs, the design variables are the twist, chord and sweep distributions.

Noise reductions are achieved through two mechanisms: phase cancellation and noise source strength reduction. Phase cancellation is accomplished either by adding blades or through sweep. The source strength dependence on propeller parameters depends on the type of noise source. The pressure perturbations due to thickness terms are proportional to relative Mach number squared, and the perturbations due to force terms are proportional to Mach number cubed. Loading noise is linearly proportional to the blade loadings though. Hence, reducing the relative Mach number through a reduction of the rotational speed or a reduction of the propeller diameter are expected to have a large effect on the noise.

The resulting optimized trade-offs are shown in tigs. 8 to 10 for the three blade types. The noise is calculated at a fixed position, $333 \mathrm{tip}$ radii away, and $30^{\circ}$ behind the plane of rotation. This angle is near the maximum of the directivity pattern, and at a distance appropriate for sideline measurements in take-off. The trade-off curves were obtained by varying the parameters in the IP, Eq. (8). The weighting value, a, was shifted from consideration of only aerodynamic performance towards consideration of noise only. For each value of the weighting parameter, the propeller geometry was re-optimized. These optimal geometries produce the curves shown.

Figure 8 shows the trade-off results for the straight blade. A simple and very effective technique for performance improvement is to increase the blade number. The blade number as shown starts at three and increases in increments of one. When either the propeller solidity is held constant, or the blade planform is held constant, the noise is decreased while the efficiency goes up. Additional blades reduce the noise by introducing phase cancellation for certain harmonics while the strengths of the remaining harmonics are constant. The efficiency is increased because of reduced tip and swirl losses. The constant solidity cases have higher efficiencies than the constant planform cases because the viscous dray is lower.

Sweeping the blade is the next most effective method shown. This trade-off starts with no sweep, and ends up with the tip swept back about $45^{\circ}$ from the initial geometry. The points along the curve represent equal increments in the weightings, but unequal changes in sweep. Sweep reduces the noise by introducing a phase shift and subsequent phase cancellation between noise from different sections of the blade. If the swept blade is concurrently retwisted to maintain the radial load distribution, the efficiency will remain nearly the same. A drawback to sweep is that for a blade with a low rotational Mach number, the amount of sweep needed for a useful reduction in noise is large. Keducing the rotational speed can reduce the noise with a small efficiency penalty. The changes shown correspond to 5 percent changes in rotational speed. The higher advance ratio results in higher blade loadings, hence greater tip and swirl losses, but there is a large decrease in the noise produced.

Shifting the loading inboard can reduce the noise, but with a sizeable efficiency penalty. The efficiency drops because the effective diameter is reduced, increasing the disk loading. The inboard shift of the loading reduces the noise by reducing the strength of the high Mach number sources, and increasing the strength of the low Mach number sources. Although hard to quantify, the loadings shown are shifted so that the peak moves from the 75 percent radius inboard to about the 60 percent radius.

A very ineffective method for reducing the noise is shown to be reducing the diameter. Changes here correspond to 5 percent reductions in diameter. The noise is reduced by the removal of the high Mach number tip sources. The remaining sources are increased in strength though, which offsets some of the reductions. The efficiency drops sharply because of the increased disk loading. This performance penalty is large and makes the method undesirable. Figure 9 shows the trade-off results for a propleted blade. The character of some of the trade-off curves has changed from the straight blade results. Most notable is the blade number trade-off which no longer shows an increase in efficiency with the decrease in noise. This curve is for a constant planform; for structural reasons, the tip thickness and chord are fixed. With increasing blade number, the constant blade planform propeller has increased viscous drag. Since the tip device is most beneficial at higher blade loadings, increasing the blade number will also reduce the efficiency gain. This reduced efficiency gain, along with the $v$ iscous drag increase, combine to reduce the efficiency of a propleted propeller. Further, 
while the total loading remains approximately constant, the additional tip thickness noise offsets the noise reductions from increased blade number.

Loading shift and diameter reduction show unfavorable trends similar to those for the straight blade. Loading shift penalizes a propleted propeller more by unloading the tip and proplet, while the proplet's effect at high loadings benefits the diameter reduction cases. Rotational speed reduction is more favorable for the propleted propeller than the straight blade propeller. As the rotational speed is reduced to decrease the noise, the loading increases. Since proplets perform better at the higher loadings, there is less efficiency loss.

Figure 10 presents the trade-offs for a biblade propeller. Again, the loading shift and diameter reduction methods have unfavorable trends. Blade number increases are for a fixed planform, to support the tip device, and so increase the viscous drag while decreasing the effectiveness of the tip device. Rotational speed reduction is more favorable than for either the straight blade or propleted propeller. This is a result of the more effective tip device retaining more of the efficiency as the loading increases. Using a rotational speed reduction, the three way bi-blade propeller has about I dBA less noise at the same efficiency as the six way constant planform straight blade propeller.

The most interesting curve is for the effect of sweep on a bi-blade propeller. After an initial decrease in efficiency with decreasing noise, the trend flattens. If the curve is extrapolated to the efficiency of a three-way constant planform straight blade propeller, the three-way bi-blade propeller would have a noise level that is $14 \mathrm{dBA}$ lower than for the straight blade propeller.

SUMNARY

A unique preliminary design tool has been developed with the ability to simultaneously optimize the performance and noise of a propeller. liy using a vortex lattice aerodynamic analysis, a subsonic compact source noise analysis, and an optimization scheme, an effective and fast preliminary design tool was created. This program was then used to investigate the noise/performance trade-offs for a straight, a propleted, and a bi-blade propeller.

While the results demonstrate the utility of such a design tool, more importantly, the relative merits of the different noise reduction techniques are identified for optinized geometries. In short, for straight blades, increasing the blade number will increase efficiency and decrease the noise. Reducing the rotational speed will also reduce the noise but will decrease the efficiency slightly. For a propleted blade, the most favorable method is to reduce the rotational speed, but again there is an efficiency penalty for noise reduction.
Finally, for a bi-blade propeller, either a rotational speed reduction or sweeping the blade will reduce noise effectively. But, with its initially greater efficiency, there is a region where the bi-blade propeller has a higher efficiency and a lower roise level than a similar straight blade propeller.

\section{REFERENCES}

1. L.K. Chang, and J.P. Sullivan, "Optimization of Propeller Blade Shape by an Analytical Method," AIAA Paper 82-1125, June 1982.

2. J.P. Sullivan, L.K. Chang, and C.J. Miller, "The Effect of Proplets and Bi-blades on the Performance and Noise of Propellers," SAE Paper 810600, April 1981.

3. V.E. Baskin, L.S. Vildgrube, Vozhdayev, Ye. S., and G.I. Maykapar, "Theory of the Lifting Airscrew, "NASA TT-F-823, February 1976.

4. L.J. Bober, and L.K. Chang, "Factors Influencing the Predicted Performance of Advanced Propeller Designs," NASA TM-82676, 1981 .

5. L.K. Chang, "The Theoretical Performance of High Efficiency Propellers," Ph.D. Thesis, Purdue University, 1980.

6. T.A. Egolf, O.L. Anderson, D.E. Edwards, and A.J. Landgrebe, "An Analysis for High Speed Propeller-Nacelle Aerodynamic Performance Prediction." Volune 1, Theory and Initial Application, NASA CR-169450, and Volume 2, User's Manual for the Computer Program, NASA CR-169451, (R79-912949-19, United Technologies Research Center; NASA Contract NAS3-20961), June 1979.

7. J.P. Sullivan, "The Effect of Blade Sweep on Propeller Performance," AIAA Paper 77-716, June 1977.

8. R.J. Jeracki, and G.A. Mitchel1, "Low and High Speed Propellers for Ceneral Aviation - Performance Potential and Recent Wind Tunnel Test Results," SAE Paper 811090, April 1981. Note: "This reference describes the tests in which the 0.566 scale data were obtained. The data contained herein have not yet been published and were obtained in a private communication with Robert J. Jeracki, Propeller Research Section, Propulsion Aerodynamics Division, NASA Lewis Research Center, Cleveland, Ohio, January 1983.

9. G.P. Succi, D.H. Munro, J.A. Zimmer, P.D. Dunbeck, E.E. Larabee, K.U. Ingard, and J.L. Kerrebrock, "Noise and Performance of Propellers for Light Aircraft," NASA CR-165732, 1981. 
10. M.V. Lowson, "The Sound Field for Singularities in Motion, " Proceedings of the Royal Society (London), Vol. A286, 1965 , pp. 559-572.

11. D.H. Munro, "The Production of Sound by Moving Objects," Ph.D. Thesis, MIT, June 1980 .

12. G.P. Succi, "Computed and Experimental Pressure Signatures from Two $1 / 4 \mathrm{Scale}$ General Aviation Propellers," NASA CR-165728, 1980.

13. G.P. Succi, "Noise and Performance of General Aviation Aircraft: A Review of the MIT Study," SAE Paper 810586, April 1981.

14. G.P. Succi, D.H. Munro, and J.A. Zimmer, "Experimental Verification of Propeller Noise Prediction," AIAA Journal, Vol. 20, No. 11, November 1982, pp. 1483-1491.
15. C.J. Miller, "Optimally Designed Propellers Constrained by Noise," Ph.D. Thesis, Purdue University, 1984 .

16. M.J.D. Powel1, "An Efficient Method for Finding the Minimum of a Function of Several Variables without Calculating Derivatives," Computer Journal, Vol. 7, No. 2, July 1964, Pp. 155-162.

17. W.I. Zangwill, W.I., "Minimizing a Function without Calculating Derivatives," Computer Journal, Vol. 10, 1967, pp. 293-296.

18. A.V. Fiacco, and G.P. McCormick, "Nonlinear Programming," New York, John Wiley \& Sons, 1968.

19. S. Goldstein, "On the Vortex Theory of Screw Propellers," Proceedings of the Royal Society of London, Series A, Vol. 123, 1929, pp. 440-465. 



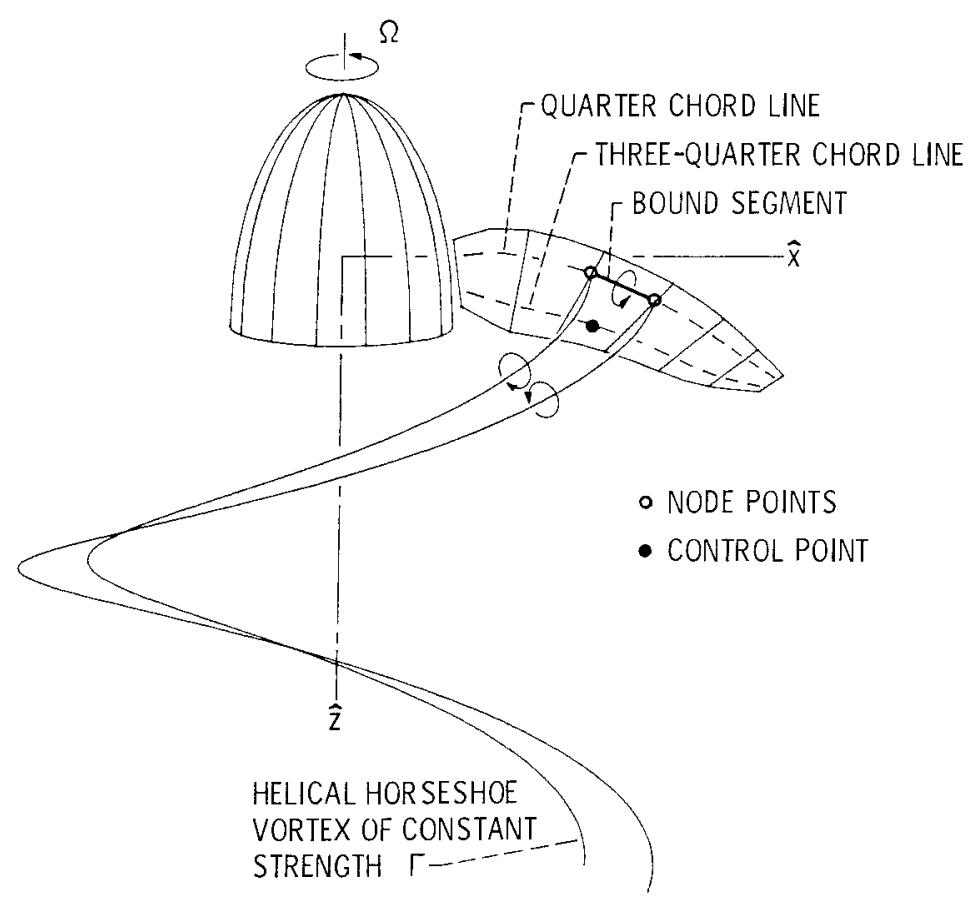

Figure 1. - Lifting line geometry.

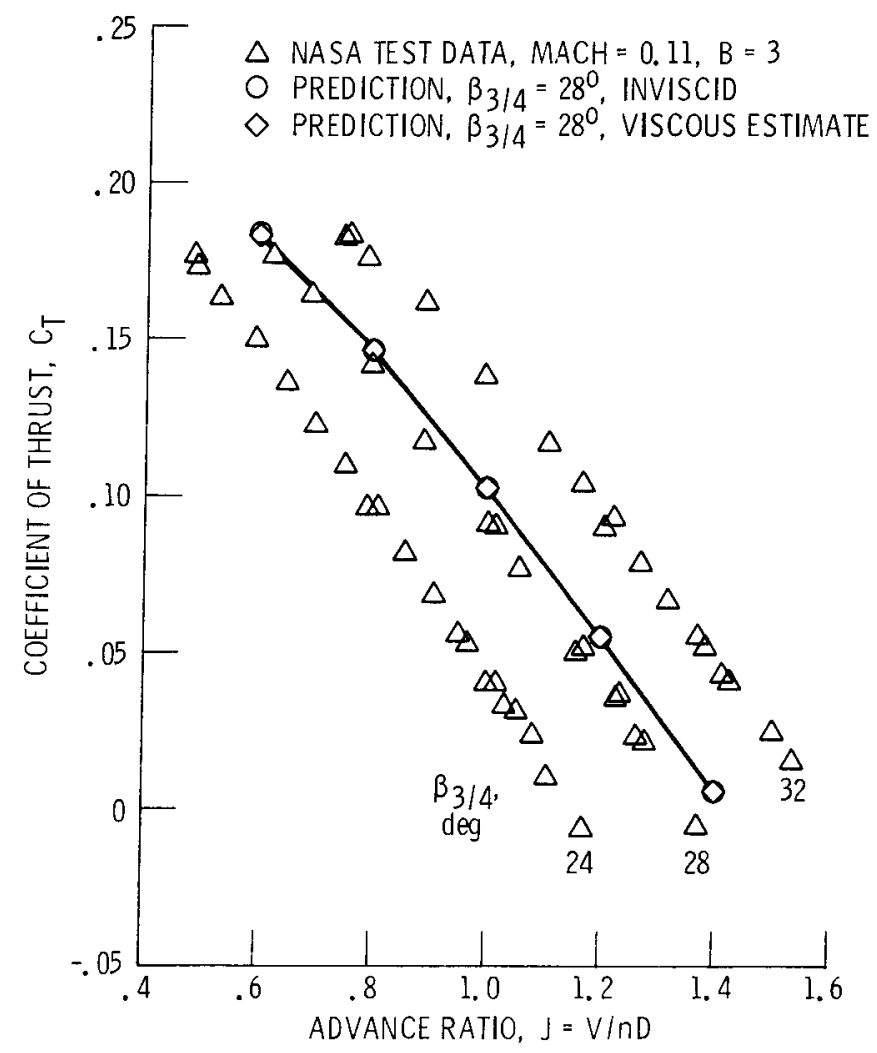

Figure 2. - Comparison of analytical and experimental thrust coefficients for the LT10282+4 propeller. Experiniental data fromi reference [9]. 


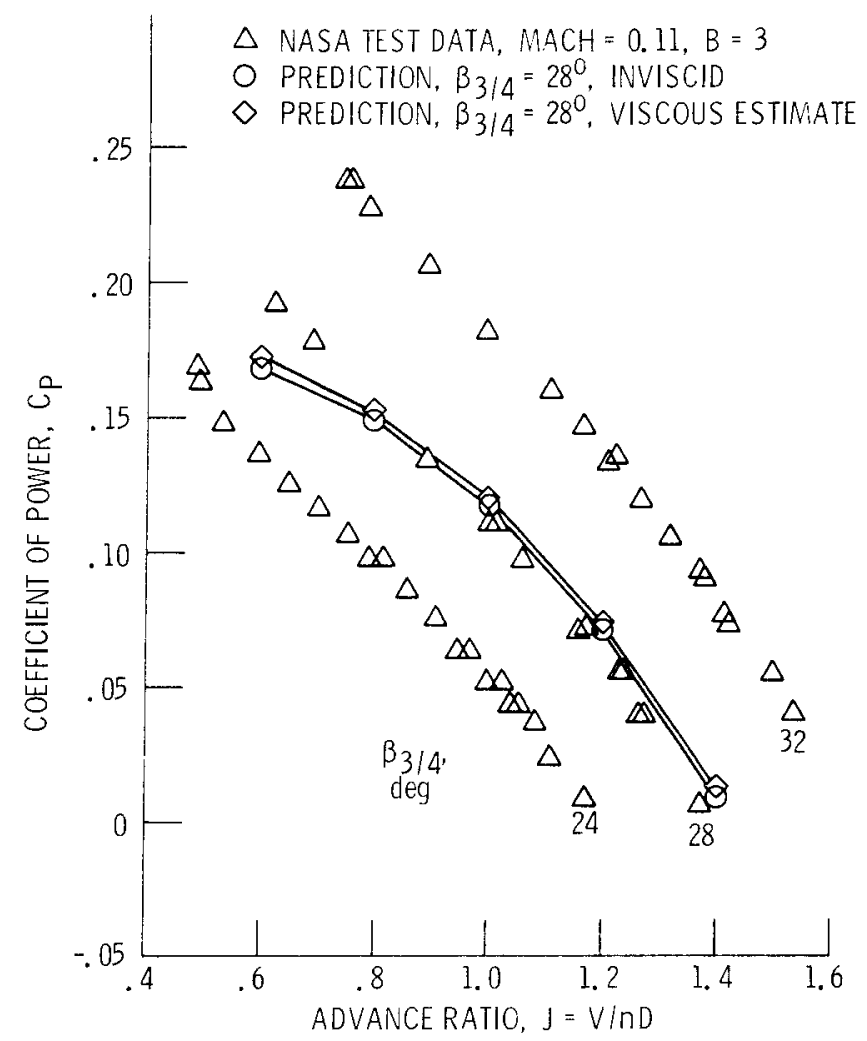

Figure 3. - Comparison of analytical and experimental power coefficients for the LTI0282+4 propeller. Experimental data from, reference [9].

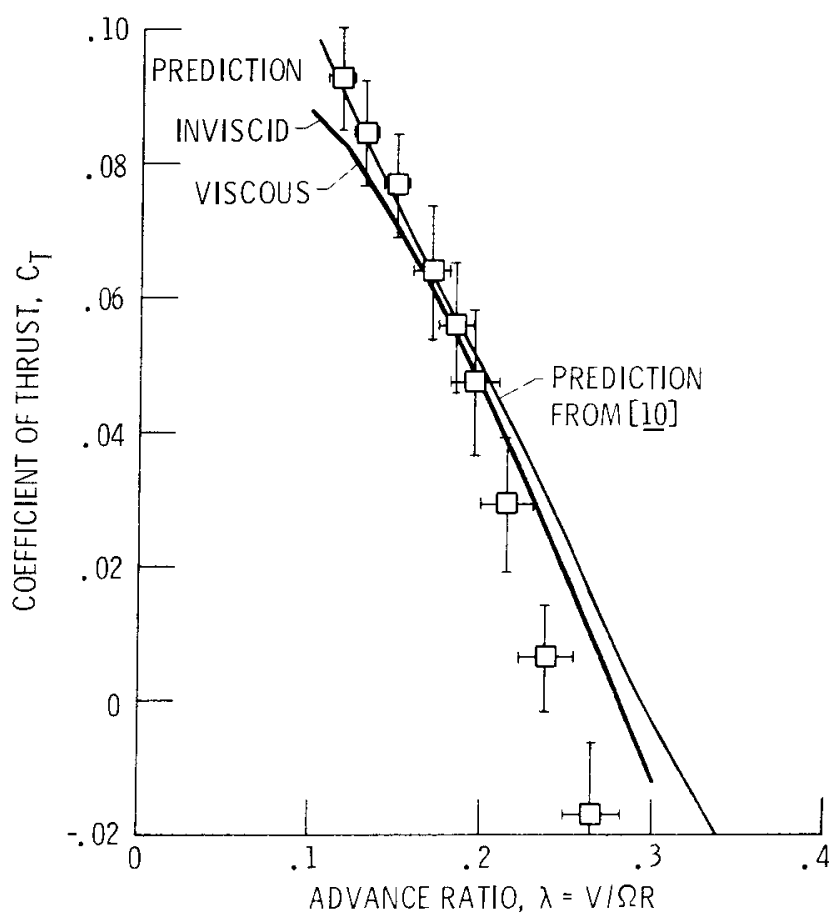

Figure 4. - Comparison of analytical and experimental thrust coefficients for the $1 C 160$ propeller. Experimental data fromi reference [10]. 


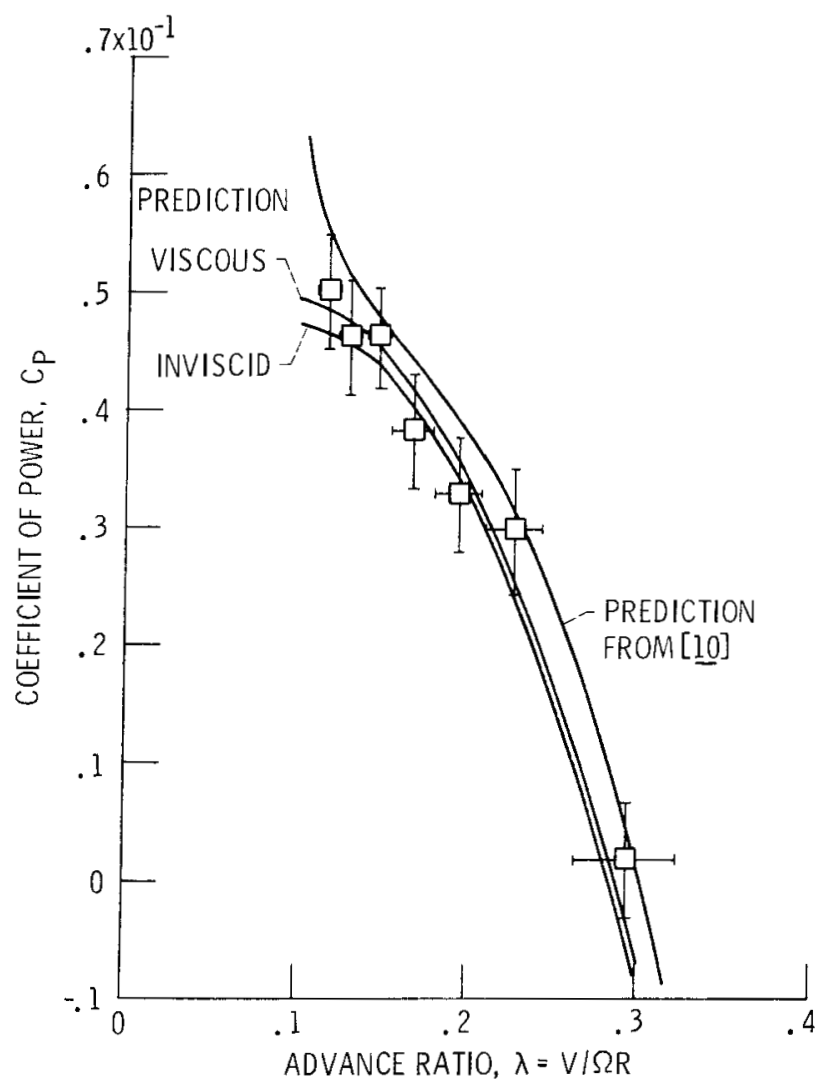

Figure 5. - Comparison of analytical and experimental power coefficients for the 1 C160 propeller. Experimental data from reference [10] reduced in value by half.

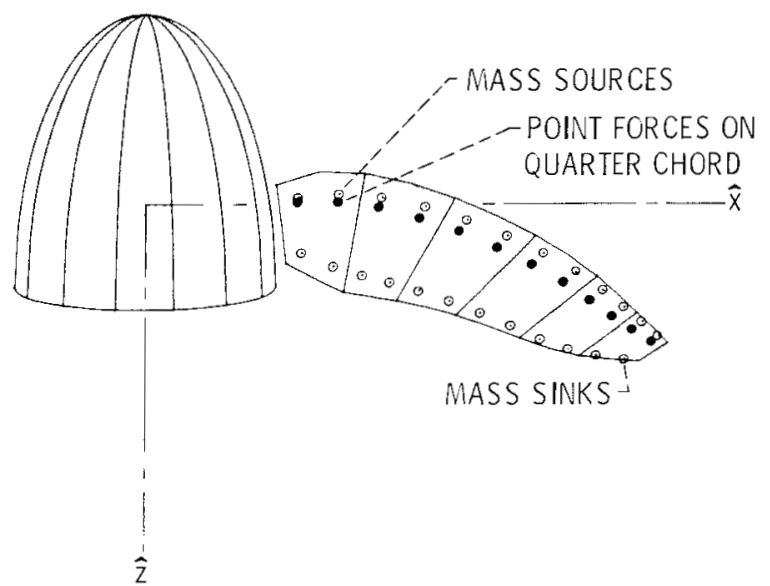

Figure 6. - Noise source locations. 


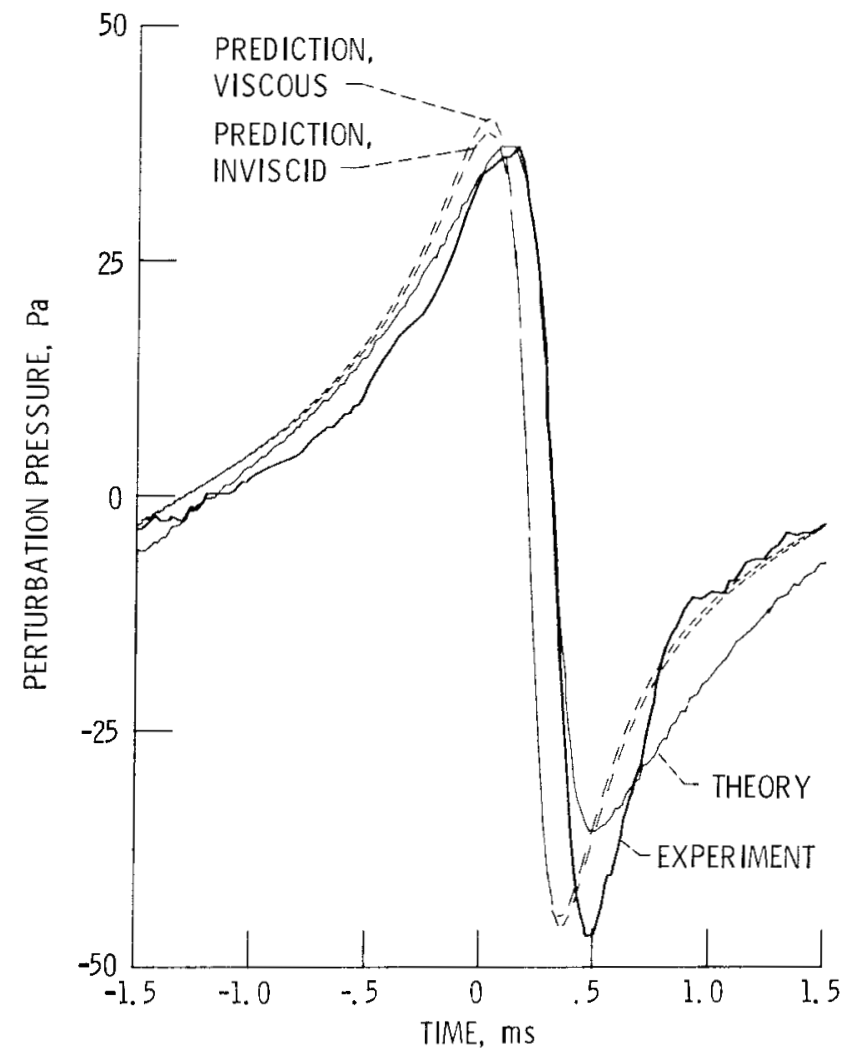

Figure 7. - Analytical and experimental comparison of near-field noise for propeller $1 \mathrm{Cl} 60$ in the plane of rotation. Experimental data and theory from references $[10,23-15]$. 


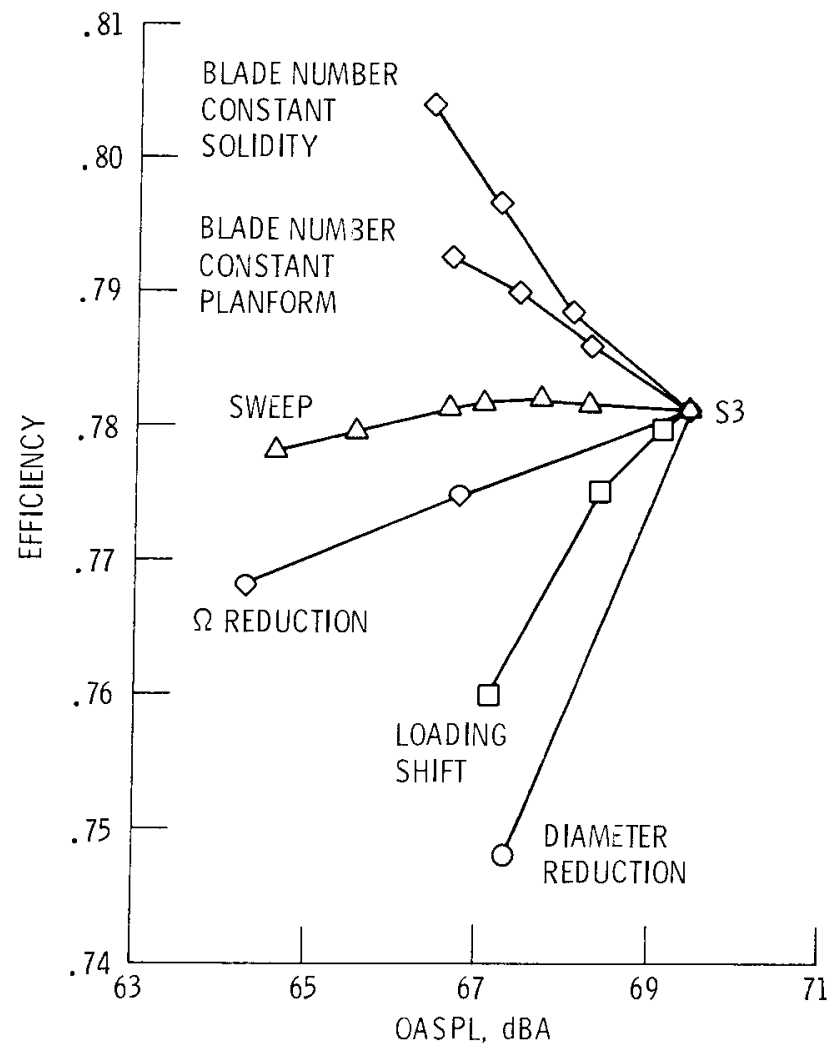

Figure 8. - Optimized noise/performance trade-offs starting from the three way straight blade (S3) baseline configuration. Design point; $J=0.8$, $c_{p}=0.154$. 


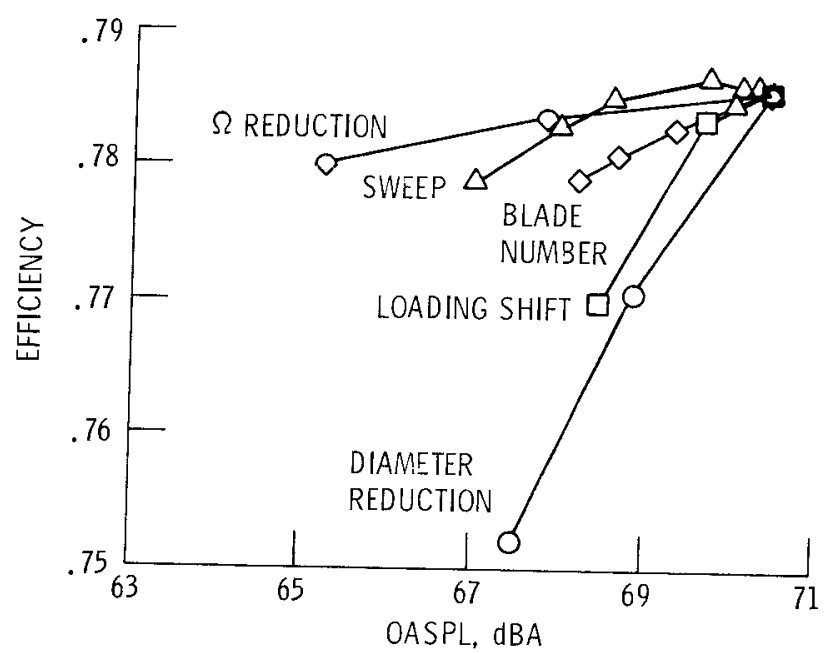

Figure 9. - Optimized noise/performance trade-offs starting from, the three way propleted (P3) baseline configuration. Design point; $J=0.8, C_{P}=0.154$.

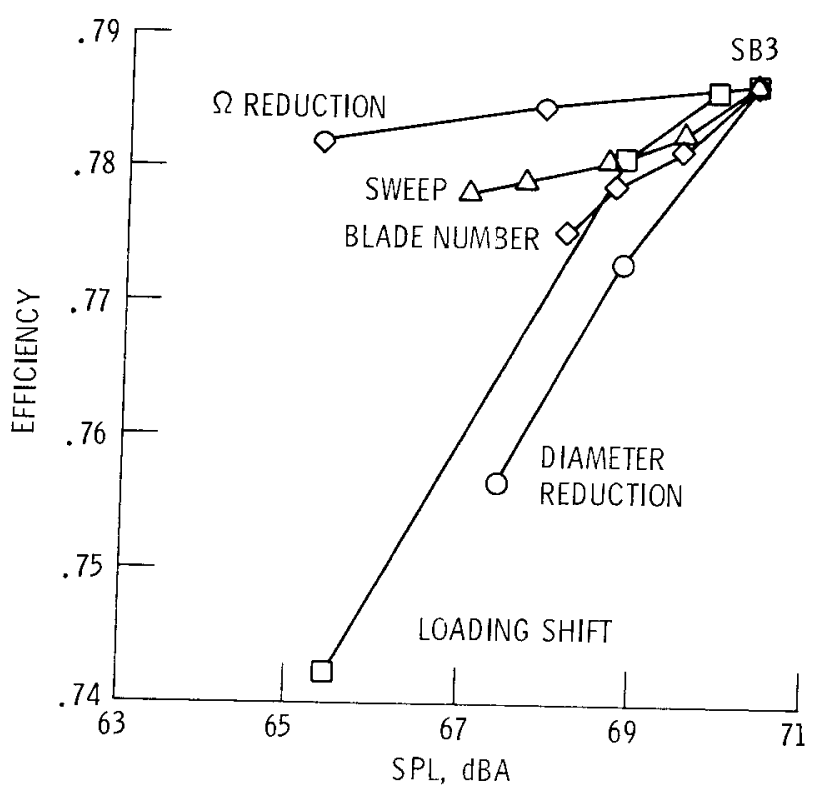

Figure 10. - Optimized noise/performance trade-offs starting from, the three way symmetrically loaded bi-blade (SB3) baseline configuration. Design point; $J=0.8, C_{p}=0.154$. 



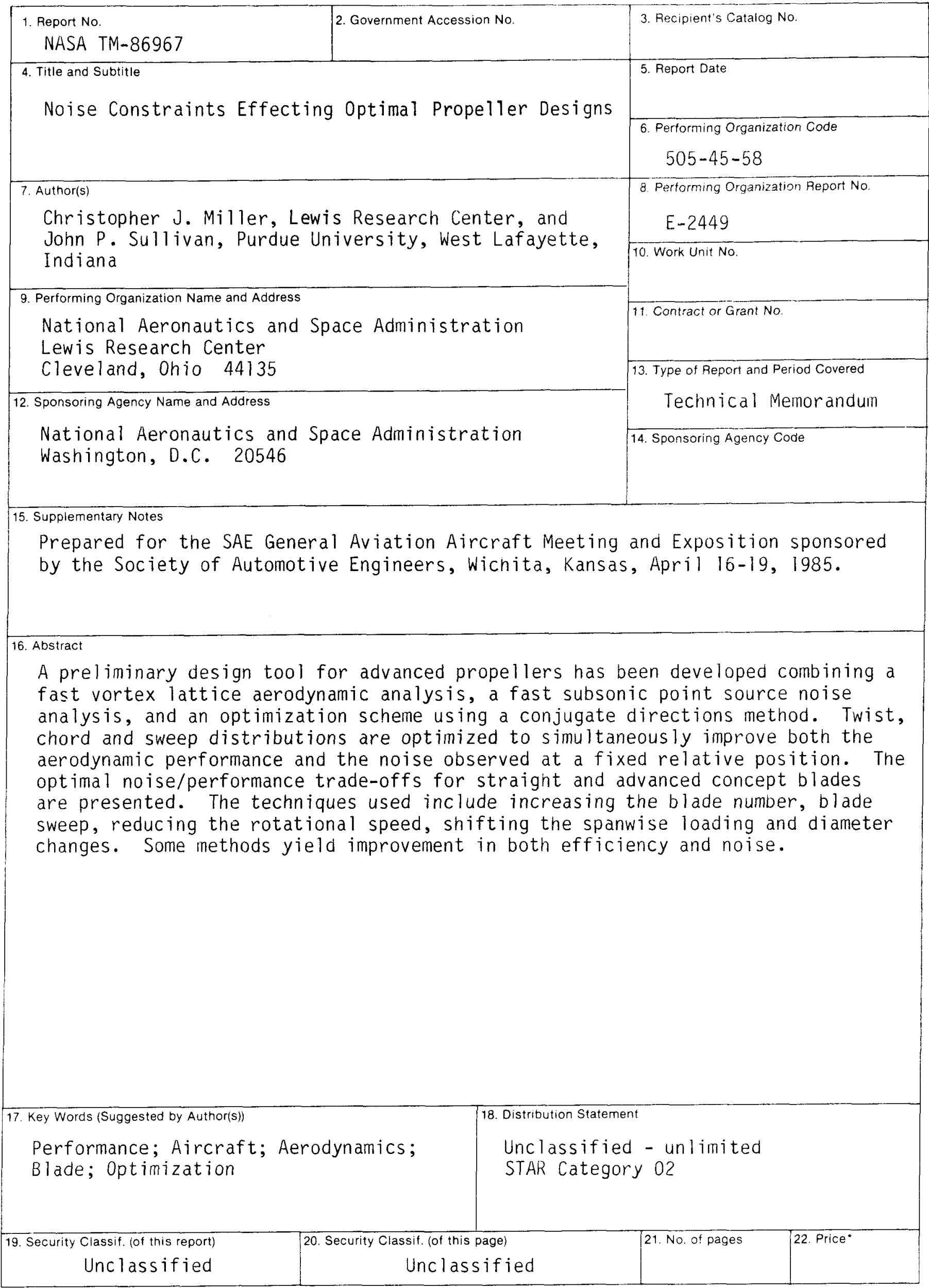

*For sale by the National Technical Information Service. Springtield, Virginia 2216 : 